\title{
A Genetic Algorithm based Approach for Multi-objective Hardware/Software Co-optimization
}

\author{
Tania Banerjee, Mohamed Gadou and Sanjay Ranka \\ Department of Computer and Information Science and Engineering \\ University of Florida, Gainesville, FL 32611 \\ Email: \{tmishra, mgadou, ranka\}@ cise.ufl.edu
}

\begin{abstract}
We develop a genetic algorithm based autotuning strategy in this paper. Autotuning is a platform independent code optimization process in which different hardware and software parameters of the code being optimized are identified and the parameter space explored to arrive at an alternative implementation that optimizes characteristics such as performance and energy consumption. The main advantage of our approach is that the number of possible compilations and executions that are explored in the configuration space is substantially smaller than exhaustive search. We demonstrate the usefulness of our approach to the underlying small matrix multiplication routines in spectral element solvers. The latter are an important class of higher order methods that are expected to be computationally intensive portion of next generation of large scale CFD simulations. Our experimental results were conducted on a variety of existing platforms as well as on gem5 simulator platform with different cache configurations. On an existing platform AMD Fusion, for example, the genetic algorithm is able obtain $34 \%$ improvement in performance and $37 \%$ reduction in energy consumption over existing versions of the code. The fact that a very small fraction of the entire configuration space needs to be explored becomes very useful as algorithmic exploration is combined with exploration of cache configuration resulting in hardware/software co-optimization. We used the micro-architectural simulator, gem5, to evaluate different cache configurations for energy and performance tradeoffs for out-of-order x86 cores at the micro-architectural level for small matrix multiplications. Our results show how genetic algorithm based autotuning strategy can come up with a close to optimal variant analyzing only about $0.25 \%$ of the exploration space.
\end{abstract}

Keywords: Spectral Element Methods, Power and Energy Evaluation, Performance Benchmarking, Exascale, Hardware/Software Co-optimization

\section{INTRODUCTION}

The increasing complexity of computer architecture has made it very difficult to program codes with optimum performance. Processor features such as vector units, multithreading and pipelining requires varied and complex optimization techniques that are rather difficult to handle either automatically by today's compilers or manually by the programmer. The problem further aggravates when we have multiple and often conflicting objectives for optimization. A practical method of optimizing these codes is autotuning. Autotuning is a method of empirical optimization in which the configuration space of a code is automatically explored in a systematic manner along with hardware parameters that impact performance and energy requirements of the algorithm, thereby finding the best values for these parameters on a given platform. Autotuning results in highly tuned codes for specific architectures and has the potential for significantly improving performance and energy requirements. Attractive though it is as an optimization technique, autotuning involves exhaustive exploration of parameter space which is time consuming. As the number of tunable parameters is increased, the exploration space increases exponentially and exhaustive search quickly becomes infeasible.

We developed a genetic algorithm (GA) based search mechanism that efficiently explores the parameter space to obtain close to optimal parameter settings for a given code. GA is a technique for solving optimization problems with or without constraints and imbibes the principles of natural selection followed in biological evolution [1]. GA has been used in a vast area of applications including multiple sequence alignment in bioinformatics [21], to find out weak links in approximate computing in computer architecture [22], and in natural language processing [23]. We use our optimization strategy on spectral element method (SEM) which is a highorder weighted residual technique that provides the geometric flexibility of finite elements, rapid convergence properties and tensor-product efficiency benefits of global structure methods [2]. They are expected to form the core computation of a large number of applications targeted for exascale computing. NEK5000, a publicly available software, is based on SEMs and is expected to be used in a variety of applications for simulating fluid flow, heat transfer and magneto hydrodynamics as well as electromagnetics [7]. CMT-Nek, an ongoing development effort at the University of Florida for compressible multiphase turbulence, is based on NEK5000. One of the most compute intensive kernels of SEM is derivative computation involving small matrix matrix multiplications and CMT-nek is expected to compute derivatives for $25-50 \%$ of its runtime.

Matrix matrix multiplication has been studied extensively in literature for large matrices. SEM, on the other hand, uses small matrices (generally of sizes between $4 \times 4$ and $16 \times 16$ ). Achieving high performance matrix multiplications for small matrices is significantly more challenging than larger matrices as the number of times data elements are copied from memory/cache to registers is relatively small. Thus the computation to data access requirements is significantly higher for smaller matrices. Our goal in this paper is to obtain high performance and energy efficient implementations of 
SEM methods for a variety of existing architectures, as well as to explore hardware/software configuration space, thereby demonstrating the efficiency of our autotuning method. In particular, we use loop unrolling and loop permutation for code transformation with the relevant software parameters being loop unroll factors and permutation sequences. We also use hardware parameters, cache size, line size and set associativity, for different cache configurations.

The main contributions of this paper are as follows:

1) We present a genetic algorithm based driver for our autotuning approach which effectively navigates through the large configuration space of the algorithms being optimized. This approach reduces the number of configurations that need to be compiled and experimentally evaluated as compared to an exhaustive approach. The approach can be used to optimize performance and/or energy and potentially a combination of these or other objectives.

2) Using the genetic algorithm based approach, we develop improved implementations of the NEK5000 SEM algorithm for multi-core architectures of traditional CPU cores for performance and energy. In particular our best codes result in implementations on AMD Fusion cores that are faster by $34 \%$ on an average as compared to the original manually tuned version of NEK5000. The amount of energy requirements are also about $37 \%$ lower on an average.

Our results also show performance and energy requirements are highly correlated for the target architectures. Thus, multi-objective optimization based on performance and energy can be greatly simplified as optimizing for performance generally results in better energy requirements and vice versa.

3) We demonstrate an efficient exploration of hardware/software design space for SEM applications using GA based search. Inclusion of hardware parameters causes the search space to become more huge. Our experiments show that GA based search can come up with close to optimal variant after evaluating only $0.25 \%$ of the search space.

The rest of the paper is described as follows. In section II, we briefly describe the spectral methods in the context of NEK5000. Section III provides our genetic algorithm based autotuning and optimization method for the underlying small matrix multiplications on traditional CPU cores and on gem5 simulator framework. Section IV provides experimental evaluation and we conclude in Section V.

\section{Spectral Element Methods And NEK5000}

NEK5000 [2] is a scientific code for modeling incompressible flows using computational fluid dynamics solver based on SEM. The code was recognized for its algorithmic quality and sustained parallel performance in 1999 with the Gordon Bell prize. It has been shown that the code scales to over a million cores.
One of the most compute intensive section of the NEK5000 code is the spectral element solver which performs matrixvector multiplication repeatedly for each spectral element. The tensor product structure of the SEMs allows the matrixvector products to be computed as a sequence of matrixmatrix multiplications, which are used to numerically compute partial derivatives of a function $U$, represented as $u_{i j k}$ in three dimensional space. The derivatives of $U$ along the Cartesian coordinates $(r, s, t)$ are given by the following equations,

$$
\begin{aligned}
& \left.\frac{\partial U}{\partial r}\right|_{i, j, k}=\sum_{l=1}^{N_{x}} A_{i l} u_{l j k} \\
& \left.\frac{\partial U}{\partial s}\right|_{i, j, k}=\sum_{l=1}^{N_{y}} B_{j l} u_{i l k} \\
& \left.\frac{\partial U}{\partial t}\right|_{i, j, k}=\sum_{l=1}^{N_{z}} C_{k l} u_{i j l}
\end{aligned}
$$

where an element in three dimensional space is assumed to have $N_{x} \times N_{y} \times N_{z}$ Gauss-Lobatto quadrature points with values of $U$ being defined at those points. $A, B, C$ are the derivative operators, of dimensions $N_{x} \times N_{x}, N_{y} \times N_{y}$ and $N_{z} \times N_{z}$ respectively. If $N_{x}=N_{y}=N_{z}=N$ then, $B=$ $C=A^{T}$. From the equations 1,2 and 3 we observe that for computing these derivatives, the fastest changing index in $U$ are the first, middle and last indices respectively. Thus the memory access pattern for matrix $U$ is different for each of the derivatives. For example, for computing partial derivatives along $r$, contiguous memory addresses are accessed, whereas for computing those along $s$ and $t$, memory is accessed in strides of $N_{x}$ and $N_{x} \times N_{y}$ addresses respectively. $N_{x}, N_{y}$, $N_{z}$ represent order of the polynomials that are fitted and values between 5 and 25 yield highly accurate results. Thus instead of large matrices, SEM requires the solution of a large number of matrix multiplications of very small matrices. We address efficient solutions for this problem in this paper.

The basic set of equivalent codes for computing partial derivatives along $r$ are given in Figure 1. We apply loop transformations to the algorithms of Figure 1 and benchmark the resulting implementations for power and performance. For computing partial derivatives along the $s$ and $t$ directions, similar sets of codes are used, except, memory access patterns for the 4loop version of partial derivatives along $s$, prevents loop fusion - so, a 4loop-fused version of code does not exist for this derivative.

We impose the following restrictions on the configuration space based on NEK5000 specifics and compiler characteristics:

1) The matrix dimensions are considered identical in each direction. Thus, $N_{x}=N_{y}=N_{z}=N$ for a three dimensional matrix.

2) Unroll factors are divisors of the matrix sizes considered. This is helpful in eliminating any residual code that would be necessary otherwise. Processing the residual code for small matrix multiplications is a non-negligible overhead. 
Algorithm: $d u d r-4 l o o p$

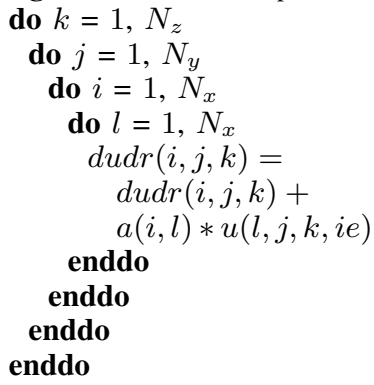

(a) 4-loop implementation
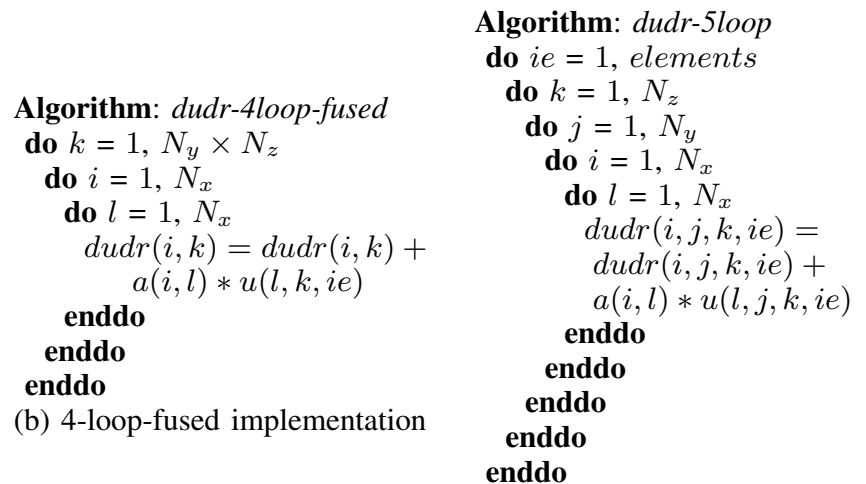

(c) 5-loop implementation

Fig. 1. Different variants of code used to compute partial derivatives of function $u$ along $r$, as shown in (a), (b) and (c). $u$ is a three dimensional discretized function defined over a spectral element. The algorithm in (d) is a top level routine that computes the partial derivatives over all spectral elements.

3) The unroll factors are limited by an amount that is likely to fill the L1 instruction cache. When the entire code fits the L1 instruction cache, performance is much better since otherwise instruction cache misses would impact performance.

Clearly, the configuration space can be further expanded to include architectural parameters such as number of ALUs, the number of instructions executed per cycle, the memory hierarchy, the number of memory ports, etc. In this paper we also explore L1 cache configurations in conjunction with algorithmic design space exploration.

\section{Genetic Algorithm based Autotuning}

\section{A. Algorithmic Exploration}

The Basic Linear Algebra Subroutines (BLAS) libraries are optimized for large matrix multiplications but do not perform well on small matrices. This was demonstrated by Shin et al in [8] as well as in our experiments on IBM BG/Q in Section IV. We use autotuning to identify the best performing as well as the most energy efficient implementation of an algorithm. The autotuning technology we use in this work is offline in nature, since it is used for building optimized libraries which are used later during runtime.

A number of projects use offline empirical autotuning frameworks: ATLAS [13], [14] is a dense linear algebra application that searches the computation space exhaustively and is used in larger matrix multiplications in BLAS and LAPACK; OSKI [15] is used for sparse matrix kernels, whereas PHiPAC [16] is an autotuning system for dense matrix multiply in C. An automatic data layout optimization was described by Sharma et al. in [17] for achieving "portable performance" on a variety of platforms. Though their method yields attractive results on computations involving stencil codes, the method is not directly applicable to matrix multiplication. Leon et al. in [12] present an analysis of how program optimization impacts power and energy consumption when the program is executed.

We develop our autotuning strategy for performance and energy using the CHiLL Framework [9] because CHiLL implements robust loop transformation procedures such as loop permutation, tiling, unroll-and-jam, data copying, iteration space splitting, fusion, and so on. For small matrixmatrix multiply, the more appropriate loop transformations are permutation and unroll-and-jam. To execute these transformations, the user specifies a script containing the necessary transformation commands. Details of how CHiLL applies these transformations are given in [9].

In our optimization strategy, we first explore the search space based on the number of elements processed in a core and the matrix sizes involved and generate variants of the matrix multiply codes for $\frac{\partial U}{\partial r}$ (Figure 1) and the corresponding codes for $\frac{\partial U}{\partial s}$ and $\frac{\partial U}{\partial t}$ using CHiLL. The search space exploration is driven by a genetic algorithm. The solution obtained using a genetic search is close to the optimal solution, and makes online evaluation possible. Though genetic algorithms have been used in a wide area of applications, it has not been applied for autotuning code transformations. The variants are created selectively in the spirit of biological evolution across generations. The variants in each generation are executed on a platform to select the fastest as well as the most energy efficient variants for that generation. Using the selected variants from the final generation, a library of routines is built which can be used in NEK5000. The library consists of the best routines for each derivative and for each matrix size for a given platform.

\section{B. Hardware Simulation Framework}

We used gem5[32] simulator and McPAT[33] power estimator for simulating the derivative computation kernels with different cache configurations and estimating power consumption. gem5 is a cycle accurate micro-architectural simulator which we use for simulating out-of-order model of x86 instruction set architecture (ISA), in system-call emulation (SE) mode and DDR3 Classic memory model. Most of the system calls in the SE mode are simulated by passing them to the operating system [34]. For estimating power consumption, McPAT takes as input a statistics file for processor utilization that is generated by gem5. The cache parameters we consider are cache size, cache line size and set associativity for L1 cache. Studying L1 cache is most relevant for this category 
of target applications involving small matrix multiplications since data size is small.

\section{Genetic Algorithm Implementation}

We describe the components and operators of GA and the overall flow below. The key components of GA in our application are as follows:

1) Individuals representing

- matrix multiplication variants created using different loop unroll factors and loop permutation sequence

- cache parameters

- a fitness value

2) Genetic operators that create new individuals from existing ones

A matrix multiplication variant is represented by each individual. An individual contains the following fields to generate the variant: a base code type, a loop permutation sequence and a set of loop unroll factors. The base code type could be 4loop, 4-loop-fused, 5-loop, or 5-loop-fused, from Figure 1. The permutation sequence is dependent upon the base code type as an example, for 4-loop-fused, the six possible permutation sequences are: "123", "132", "213", "231", "312" and "321". The possible unroll factors are set to divisors of the iteration space. Section IV-B5 discusses the different values we set for the cache parameters and the fact that certain combinations of these values are inadmissible. Therefore, instead of having separate fields for each cache parameter in the individual, we add a single parameter to each individual that represents a combination of cache parameters.

Example: Figure 2 (a) shows an individual that represents a 5-loop implementation. The permutation sequence for the matrix multiplication variant is " 14523 ", and the unroll factors for the 5 loops (before permutation is applied) are 10, 2, 5, 10,10 respectively. The last field represents cache parameter combination for this individual which is 3 and represents the setting of $2 \mathrm{~KB}, 64 \mathrm{~B}$ and 8 for cache size, line size and associativity respectively. 5loop 10 2 2 “14523" 5 10 10 10 3

(a)

\begin{tabular}{|c|c|c|c|c|c|c|c|}
\hline 5loop & 10 & 2 & “14523" & 5 & 10 & 10 & 3 \\
\hline \multicolumn{5}{|c|}{$\mathrm{P}$} & \multicolumn{3}{|c|}{ Random mutation } \\
\hline 5loop & 10 & 2 & “14523" & 2 & 10 & 10 & 3 \\
\hline
\end{tabular}

(b)
Fig. 2. (a) Example of an individual having seven characteristics. (b) Example of mutation operation. $\mathrm{P}$ is mutated to $\mathrm{Q}$.

A fitness value is assigned to each individual as a measure of the performance or energy consumption of the matrix multiplication variant represented by that individual. Depending upon the priority of optimization, fitness values could be metrics such as $E$ (energy), $E D$ (energy $\times$ delay), $E D^{2}$ (energy $\times$ delay $\times$ delay), or $T$ (total execution time).

A large number of individuals constitute a population. Two individuals are combined to create a child that belongs to the next generation. The initial population (or the first generation), consists of random individuals and also a target individual that represents the original NEK5000 matrix multiplication

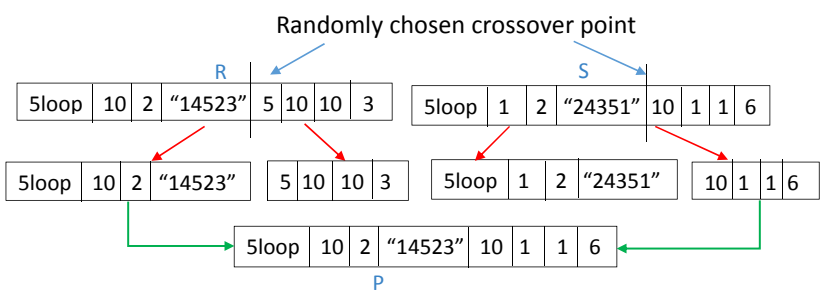

Fig. 3. An example demonstrating single point crossover in our implementation. Crossover is an operation in which complementary material from the two individuals are combined to form a new individual.

algorithm. The target individual is useful as a seed and also to set a benchmark on the improvement in fitness value obtained in a generation. The next generation of individuals is created using operators on pairs of individuals in the current generation of population. The following operators are used:

1) Mutation Operator: A mutation operator on an individual $P$ results in a new individual $Q$ by randomly altering one or more characteristics of $P$. For example, as shown in Figure 2 (b), an unroll factor in $P$ may be mutated from 5 to 2 to form a new individual $Q$.

2) Crossover Operator: A crossover operator combines a pair of individuals $R$ and $S$ to create a child $P$. In our work, we begin with a single point crossover. The crossover point is randomly chosen and complementary material from $R$ and $S$ are merged to form $P$. This is shown in Figure 3.

Additionally, our genetic algorithm uses the best solutions derived so far to guide the mutation and crossover. This improves the rate of convergence and reduces the number of generations required. This is done in two ways. Firstly, we include an individual that represents the NEK5000 matrix multiplication in the first generation. This ensures that the results we get are at least as good as the NEK5000 algorithm. Secondly, we use knowledge-based non-uniform crossover by biasing the inheritance of permutation sequence from the better performing parent. This is based on the observation that the loop permutation sequence has a greater impact on performance than the unroll factors. Knowledge based nonuniform crossover was introduced by H. Maini et al. in [18]. Random mutation and crossover may result in incompatibilities in the individual characteristics and is fixed using context based knowledge into the system. An example of such an incompatibility is a 5-loop base code getting a permutation sequence "132".

The overall GA flow in our implementation, for a given matrix size $N \times N \times N$ and a given partial derivative (one among $\left.\frac{\partial U}{\partial r}, \frac{\partial U}{\partial s}, \frac{\partial U}{\partial t}\right)$, is depicted by the flowchart in Figure 4. The first step is to generate an initial population of $n$ individuals. For each individual, its matrix multiplication algorithm is created, compiled and run to obtain and assign a fitness value to the individual. The individuals in a population are then sorted based on their fitness values starting with the best individual having the least runtime. The fitness value of the best individual is printed and a stopping condition is evaluated. If the stopping condition is not satisfied, a new generation of population is created from pairs of individuals in the current 


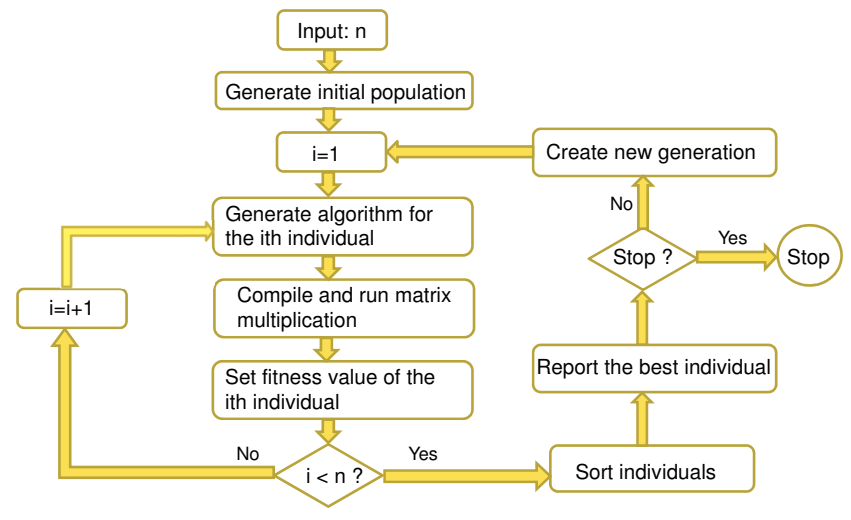

Fig. 4. Flowchart showing how genetic algorithm is integrated with autotuning

generation using the GA operators. Each individual in the new generation undergoes the same process for fitness assessment and the whole loop repeats. Since the minimum runtime is not known in advance, we break the loop if the last three generations result in the same best individual, or if a pre-defined maximum number of iterations is reached. Since processing an individual in a generation is completely independent by itself, the GA flow was parallelized by assigning the individuals in a generation to different cores.

\section{Related Work}

Closely related to our work is [7] by J. Shin et al., which also uses CHiLL for optimizing performance for small matrix multiplications in NEK5000. The main difference in our work with [7] is that we use a genetic algorithm based driver in our work, that significantly cuts the number of matrix multiplication variants to be evaluated compared to [7] which uses exhaustive search with specialization. Our work is also quite closely related to the work by Tiwari et al. in [5] where the authors combine CHiLL with Active Harmony [19] to create a scalable autotuning framework. Active Harmony provides the parallel search backend for the equivalent implementations generated by CHiLL. While a parallel search mechanism makes the search faster, it is nevertheless exhaustive and it would require us to evaluate millions of equivalent implementations which would still consume a significant amount of time [7]. In our case we combine CHiLL with our own genetic driver to create an autotuning framework, that is fast and efficient.

The primary focus of hardware/software co-design are system level synthesis and platform configuration. System level synthesis maps task level components of a software application on heterogeneous hardware/software architecture thereby satisfying contradictory constraints and achieving optimization goals [35], [36]. Platform configuration involves tuning architectural parameters by exploring the design space [37], [38], [39], and our work belongs to this category. Givargis et. al in [37] describes a system level exploration technique involving power/performance tradeoffs. In this technique the search space is divided into clusters. Each cluster is exhaus- tively searched for local Pareto-optimal solutions. The authors here suggest the use of genetic algorithm if the exhaustive search gets too slow. Givargis et. al in [38] use a simulation framework with the exploration framework of [37]. Our work is GA based and we use simulator feedback to evaluate configurations. Ascia et al. in [39] introduce a genetic algorithm based approach for exploration of architectural parameters of the processor, memory subsystem and bus. In our paper, we demonstrate an efficient and novel hardware/software codesign strategy for implementing derivative computation kernels and the cache sub-system.

\section{EXPERIMENTAL RESULTS}

We performed our experiments using autotuning based on exhaustive search and genetic algorithms on four CPU platforms - IBM BG/Q, AMD Opteron 6378, AMD Fusion A105800K and Intel Xeon E5-2670 Sandy Bridge architecture. A description of the architectures are included in Section IV-A and the results on these architectures are presented in Section IV-B.

\section{A. Platforms}

a) IBM BlueGene/Q: These experiments were performed on Cetus which is a IBM BG/Q platform at the Argonne National Laboratory (ANL). Cetus has 1024 nodes, with each node having 16 cores, each core being a PowerPC A2 processor operating at $1.6 \mathrm{GHz}$ clock speed. A node board of processors consists of 32 nodes and power is measured on a node board basis [10]. IBM BG/Q provides Environmental Monitoring (EMON) API that enables power measurement by the user as a user application is run. The EMON APIs were further made usable by the MonEQ [11] wrapper library that makes it possible to read individual voltage and current data points. BG/Q has seven power domains [12] of which core logic power and memory power are the two domains that respond to algorithmic changes in our experiments, and account for about $75 \%$ of the total power. Therefore, we measure and use the total power consumed in these two domains only. The power consumed in the remaining five domains is about the same for all the experiments. To compile the variants we use BG/Q cross compiler mpixlf77 and mpixlc, with optimization options "-O3 -qhot -qstrict". To measure performance on IBM BG/Q, we executed each matrix multiply variant on 32 MPI ranks and measured the average runtime.

b) AMD Opteron: These experiments were run on the HiPerGator infrastructure at the University of Florida which is a system of interconnected Dell C6145 nodes. Each node has four AMD Opteron 6378 CPUs, and each CPU consists of 16 cores operating at $2.4 \mathrm{GHz}$. On Opteron 6378 , there are no APIs that could give the power consumption when a specific routine is executed. So we use this platform for benchmarking performance only. The variants are compiled using gfortran with optimization flag "-O3".

c) AMD Fusion: These experiments were run at the Sandia National Laboratory on teller which is a 104 node 
commodity cluster, where each node is a AMD Fusion A10$5800 \mathrm{~K}$ processor operating at $3.8 \mathrm{Ghz}$. Component level power measurement on this commodity cluster is made possible by a device called PowerInsight [6]. The variants are compiled with GNU MPI compilers, using optimization flag "-O3".

d) Intel Xeon: These experiments were run on the cab system at Lawrence Livermore National Laboratory (LLNL). $\mathrm{Cab}$ is a 1296 node cluster, where each node has 2 Intel Xeon E5-2670 CPUs with 8 cores each. The CPU speed is $2.6 \mathrm{GHz}$, with a maximum turbo frequency of $3.3 \mathrm{GHz}$. We used Intel's Running Average Power Limit [29] (RAPL) to measure the average power consumption as well as to set an upper bound on the average power consumption over an interval of time. Applying a hardware-enforced power bound is a far more attractive option than using Dynamic Voltage and Frequency Scaling (DVFS) [40] because the mechanism of enforcing the power bound is flexible enough that the user can simply specify the bound and the power consumption of the program is controlled dynamically by the operating system either by activating specific frequencies or by turning off certain cores. RAPL uses performance counters and energy models to estimate the average power consumption and is quite accurate in its estimation [24], [25], [26], [27], [28]. The RAPL functionality is implemented via model-specific registers (MSRs). These registers are accessible on $c a b$ via a kernel module msr-safe [30] which allows safe access to the MSRs. The msr-safe kernel module on the other hand is accessible via a user friendly interface, the libmsr [31] library, which we use extensively in our work.

\section{B. Results}

We present results from two sets of experiments. The first set exhaustively covers the search space for a representative matrix size to identify the most efficient code variant for that matrix size. The number of combinations was too large to apply exhaustive search to all relevant matrix sizes. The second set of experiments applies genetic algorithms to derive the efficient matrix multiplication variants for a number of possible matrix sizes. While using genetic algorithms, population size of the generations was set to 100 .

\section{1) $I B M B G / Q$ :}

a) Exhaustive search: We set the matrix size for the exhaustive runs to $N_{x}=N_{y}=N_{z}=10$. The total number of variants that we experimented with were approximately 100,000 (this is based on variable loop unroll factors and number of loop permutations) for each matrix size. Each variant is run 500 times and the measurements averaged to obtain a runtime measure of that variant.

Figure 5 shows the performance of the best variation of matrix multiplication algorithm for the various base codes for each of the three derivatives. We compare our results with the corresponding NEK5000 kernels and also with the work in [7] which we hereafter refer to as SMC. The y-axis in these as well as the other performance graphs represent normalized runtimes of the matrix multiplication algorithms, and is calculated by dividing the actual runtime of an algorithm
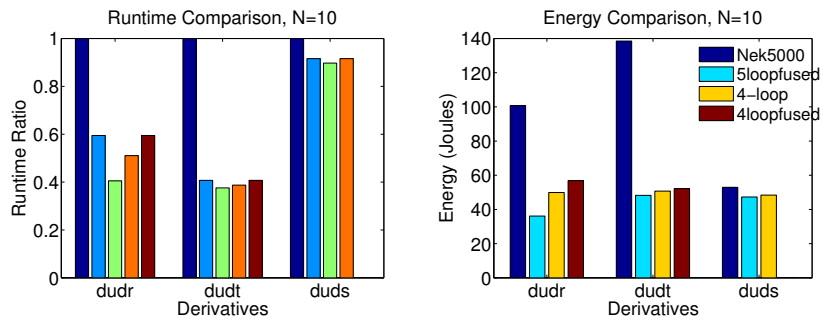

Fig. 5. Performance and energy comparison on IBM BG/Q for matrix size $10 \times 10 \times 10$. There is a $53.7 \%$ improvement in both runtime and energy on an average over the three derivatives, using autotuning with exhaustive search.

by the runtime of NEK5000 kernel for the same derivative computation. The 4loop version give us the best performance for $N=10$ on the BG/Q architecture. The performance relative to NEK5000 implementation of the derivatives is about 59.4, 62.4 and 26.2 percent faster for $d u d r$, dudt and $d u d s$ respectively, and the aggregate improvement was $54.3 \%$ (or about 2 times) for $N=10$. Aggregate improvement is computed as $\left(T_{n e k}-T_{a u t o}\right) / T_{n e k} \times 100$, where $T_{n e k}$ is the total time taken to compute $d u d r$, dudt and $d u d s$ using the existing NEK5000 algorithms, whereas $T_{\text {auto }}$ is the total time taken to compute the three derivatives using autotuned matrix multiplication algorithm. If we replace $T_{n e k}$ with $T_{s m c}$ in the equation for aggregate improvement, we get the corresponding aggregate improvement with respect to SMC, which in our experiments were $14.5 \%$ for $N=10$.

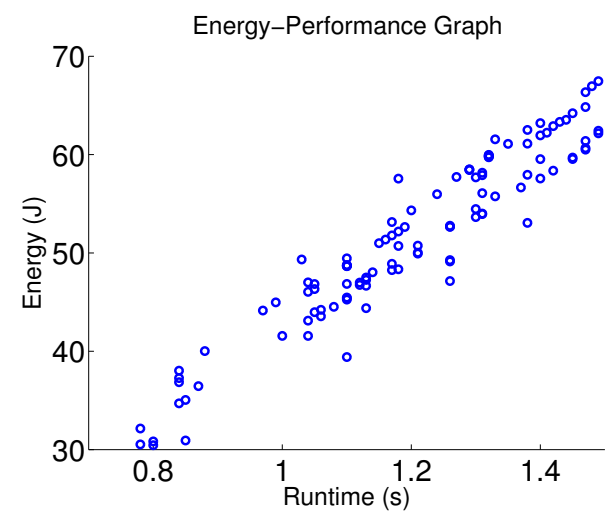

Fig. 6. Total energy versus runtime graph on IBM BG/Q. Each point on the graph represents a matrix multiplication variant and its location is determined by its performance and energy consumption.

Figure 5 also shows the total energy requirements of the best variants in terms of energy consumption per node. Starting from booting a partition, it takes about 10 minutes for each code variant enabled with power measuring MonEQ APIs, to report the power numbers. Therefore, we did not measure power for all the variants, but for a small fraction of the high performing variants based on execution time, and we studied their power and energy requirements. To obtain the total power, we first ran a variant on 32 nodes (and 32 cores per node), captured the node board power, divided it by 32 to get the power per node.Total energy is obtained by multiplying the 
total power consumed by the variant and the time taken to run that variant. The energy optimal variants are either same as the performance optimal variants or have high performance, very close to the performance optimal variants used in Figure 5.

We also compared the performance and total energy requirements (memory and core) of the different variants for dudt-4loop-fused base code and the results are presented in Figure 6. The energy versus performance results show that energy is highly correlated with performance.

Further, the three derivatives have different characteristics of the best variant in terms of permutation sequence and unroll factors. Thus, the optimal matrix multiplication implementation depends on the derivative to be computed.

b) Genetic Algorithm Evaluation: At the time of performing the experiments, ROSE was not supported on IBM $\mathrm{BG} / \mathrm{Q}$, hence to evaluate the GA setup we used a static approach in which the runtime of a variant is looked up from a table constructed using runtimes from the exhaustive experiments. Figure 7 shows a comparison of the runtimes for matrix sizes $10 \times 10 \times 10$.The first column lists the derivatives, the second column shows the runtime for NEK5000 implementation of the corresponding derivative, columns third to fifth show the statistics for the exhaustive run, including the runtime in seconds, the number of variants analyzed and the improvement percent over the NEK5000 implementation. The columns sixth to eighth give the corresponding numbers for the GA based autotuning approach of this paper. Thus on an average over the three derivatives, genetic algorithms found a variant that performed within $1.6 \%$ of the optimal performance (computed from data in 3rd and 6th columns in Figure 7) using about $1.15 \%$ of the total variants as analyzed in the exhaustive approach (computed from data in the 4th and 7th columns of the figure).

\begin{tabular}{|c|l|l|l|l|l|l|l|}
\hline Derivatives & \multirow{2}{*}{$\begin{array}{l}\text { NEK5000 } \\
\text { Time }\end{array}$} & & \multicolumn{3}{|c|}{ Exhaustive Autotuning } & \multicolumn{3}{|c|}{ GA based Autotuning } \\
\cline { 3 - 8 } & Time & Variants & $\begin{array}{l}\text { \%impro- } \\
\text { vement }\end{array}$ & Time & Variants & $\begin{array}{l}\text { \%impro- } \\
\text { vement }\end{array}$ \\
\hline dudr & 1.9 & 0.77 & 32652 & 59 & 0.78 & 344 & 59 \\
\hline duds & 1.07 & 0.79 & 32412 & 26 & 0.79 & 295 & 26 \\
\hline dudt & 2.60 & 0.98 & 32652 & 62 & 1.01 & 485 & 61 \\
\hline
\end{tabular}

Fig. 7. Comparison of compute times on IBM BG/Q for matrix size $10 \times 10 \times 10$, between autotuning based on exhaustive search and GA based autotuning. Only about $1 \%$ of the variants are analyzed by the GA, while performance of best variant found by the GA based approach is comparable to the optimal version (found using exhaustive approach). Time unit is second.

\section{2) AMD Opteron:}

a) Exhaustive search: Figure 8 (a) shows the performance of the best variation for each of the various base codes for the three different derivatives and matrix size $N=10$. The aggregate improvement in performance with respect to NEK5000 was $43 \%$ while the aggregate improvement in performance with respect to SMC was 5.3\%.

b) Genetic Algorithm Evaluation: Figure 8(b) shows a comparison of the total runtime for computing the three derivatives $d u d r$, duds, and $d u d t$ using NEK5000 algorithm and using the best variants found by the genetic algorithm, for different matrix sizes.The setup of this experiment was
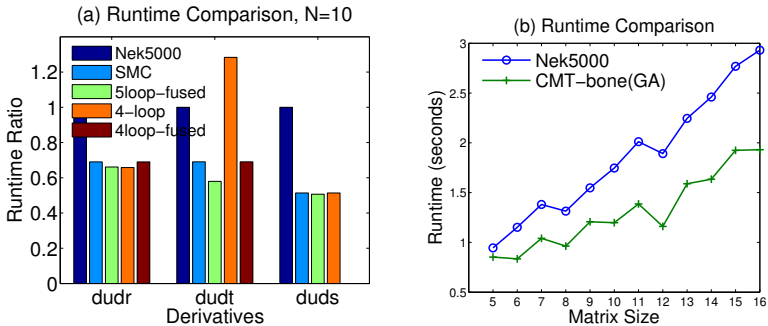

Fig. 8. Performance of matrix-matrix multiply kernels on Opteron. Graph (a) shows the performance ratio of the best variants using exhaustive search for matrix size $10 \times 10 \times 10$. Graph (b) shows performance of autotuning using genetic algorithms over matrix sizes between $5 \times 5 \times 5$ through $16 \times 16 \times 16$.

\begin{tabular}{|c|l|l|l|l|l|l|l|}
\hline Derivatives & \multirow{2}{*}{$\begin{array}{l}\text { NEK5000 } \\
\text { Time }\end{array}$} & \multicolumn{3}{|c|}{ Exhaustive Autotuning } & \multicolumn{3}{|c|}{ GA based Autotuning } \\
\cline { 3 - 8 } & & Time & Variants & $\begin{array}{l}\text { \%impro- } \\
\text { vement }\end{array}$ & Time & Variants & $\begin{array}{l}\text { \%impro- } \\
\text { vement }\end{array}$ \\
\hline dudr & 0.62 & 0.39 & 32652 & 37 & 0.41 & 444 & 34 \\
\hline duds & 0.31 & 0.18 & 32412 & 42 & 0.2 & 393 & 35 \\
\hline dudt & 0.88 & 0.45 & 32652 & 49 & 0.45 & 446 & 49 \\
\hline
\end{tabular}

Fig. 9. Comparison of compute times on AMD Opteron for matrix size $10 \times 10 \times 10$. GA based approach analyzes a small fraction of the total variants analyzed by exhaustive search, while performance of the best variant found by GA is comparable to that found using exhaustive search. Time unit is second.

such that the workload for each $N$ is about the same, thus when $N$ is large (small), we compute a smaller (larger) number of matrix multiplications. From this graph, we find that the performance of our algorithms is better than that of the NEK5000 kernels by $9.7 \%$ for $N=5$ up to $39 \%$ for $N=12$, with an average improvement of $28 \%$. The total number of variants analyzed was less than 600 for each $N$.The best algorithm found for each matrix size has different permutation and unroll characteristics, which points to the fact that the high performing algorithms have different characteristics for different matrix size $N$.

Figure 9 shows a comparison of run times of various matrix multiplication algorithms for the three derivatives $d u d r, d u d s$, and $d u d t$, for matrix size $10 \times 10 \times 10$. The genetic algorithm finds variants which perform within $4 \%$ of the optimal performance (see 3rd and 6th columns) on an average over the three derivatives and the number of variants analyzed was about $1.31 \%$ of the total variants analyzed in the exhaustive search (see 4th and 7th columns).

\section{3) AMD Fusion:}

a) Exhaustive search versus genetic algorithm driven search: Since running an exhaustive search is time consuming, we completed an exhaustive analysis only for derivative $\frac{\partial U}{\partial r}$ for matrix size $10 \times 10 \times 10$ with the idea of validating the

\begin{tabular}{|c|l|l|l|l|l|l|l|}
\hline Derivatives & NEK5000 & \multicolumn{3}{|c|}{ Exhaustive Autotuning } & \multicolumn{2}{|c|}{ GA based Autotuning } \\
\cline { 3 - 8 } & Time & Time & Variants & $\begin{array}{l}\text { \%impro- } \\
\text { vement }\end{array}$ & Time & Variants & $\begin{array}{c}\text { \%impro- } \\
\text { vement }\end{array}$ \\
\hline dudr & 1.36 & 0.95 & 32652 & 30 & 0.95 & 485 & 30 \\
\hline
\end{tabular}

Fig. 10. Comparison of compute times in seconds for $\frac{\partial U}{\partial r}$ on AMD Fusion. 

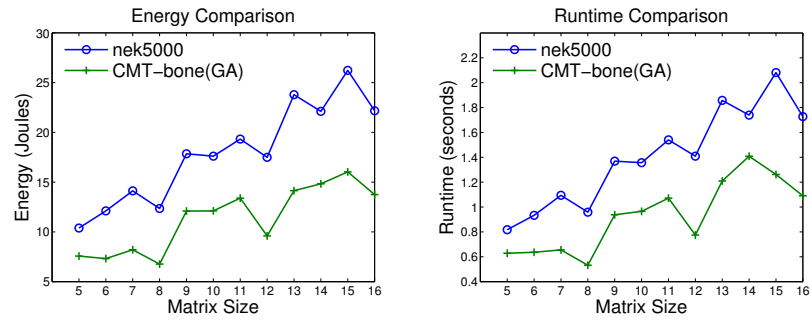

Fig. 11. Comparison of energy consumption for matrix sizes between $5 \times 5 \times 5$ through $16 \times 16 \times 16$ on AMD Fusion. The best improvement is observed for matrix sizes $8 \times 8 \times 8$ and $12 \times 12 \times 12$ which is about $45 \%$ for performance and energy.

corresponding best version obtained using genetic algorithms. Figure 10 shows a comparison of run times. The genetic algorithm found the same optimal variant using about $1.49 \%$ of the total variants that were analyzed by the exhaustive approach.

Figure 11 shows the total energy consumption and total runtime of the best variants obtained using genetic algorithms compared to the NEK5000 implementation. The fitness function for the energy experiment was total energy, while that for the performance experiment was runtime. The plots of the best variants are compared to that of our NEK5000 benchmark. Both energy and runtime are summed for all the three derivatives. The improvement in energy consumption was between $27 \%$ for matrix size $N=5$ and $45 \%$ for matrix sizes $N=8$ and $N=12$, with an average improvement of $37 \%$. The improvement in performance was between $23 \%$ and $45 \%$ with an average improvement of $34 \%$. For most matrix sizes, the highest energy efficient variant was also the one with the best performance. The CPU and memory power in Watts for the partial derivatives are about the same across different matrix sizes, and differences in energy is largely due to differences in runtime.

\begin{tabular}{|c|l|l|l|l|l|l|l|}
\hline Derivatives & NEK5000 & \multicolumn{2}{|c|}{ Exhaustive Autotuning } & \multicolumn{3}{|c|}{ GA based Autotuning } \\
\cline { 3 - 7 } & Time & Time & Variants & $\begin{array}{l}\text { \%impro- } \\
\text { vement }\end{array}$ & Time & Variants & $\begin{array}{c}\text { \%impro- } \\
\text { vement }\end{array}$ \\
\hline dudr & 18.36 & 11.95 & 5016 & 35 & 11.97 & 120 & 34.8 \\
\hline
\end{tabular}

Fig. 12. Comparison of compute times of $\frac{\partial U}{\partial r}$ on Intel platform. Power bound set to $50 \mathrm{~W}$ and each variant is run 1000 times. Exhaustive autotuning finds optimal variant after analyzing 5016 different 4loop and 4loop-fused implementations of the derivative (takes 3 days to run), whereas GA based autotuning finds a variant very close to the optimal after analyzing only 120 variants (takes 1 hour 30 minutes to run.

4) Intel Xeon: We now present a comparative study of exhaustive search versus GA driven search for the computation of $\frac{\partial U}{\partial r}$ for a $16 \times 16 \times 16$ element on $c a b$. Figure 12 shows a comparison of the optimal version of the algorithm (found using exhaustive search) compared to the best variant identified by using GA driven search. From this table we see that while the optimal version gives us an improvement of $35 \%$ over the native implementation, the version identified by GA gives us an improvement of $34.8 \%$ over the native implementation.
Thus, the GA version found a near optimal variant after analyzing only $2.4 \%$ of the variants that are analyzed by the exhaustive search. It is to be noted that the power bound for this experiment was set at 50W. Also the time reported here is the total time to run each variant 1000 times to ensure that the program runs for at least 10 seconds for stable results with power bounds. We studied the 4loop and the 4loop-fused versions of the algorithms here which reduces the number of variants to 5016 .

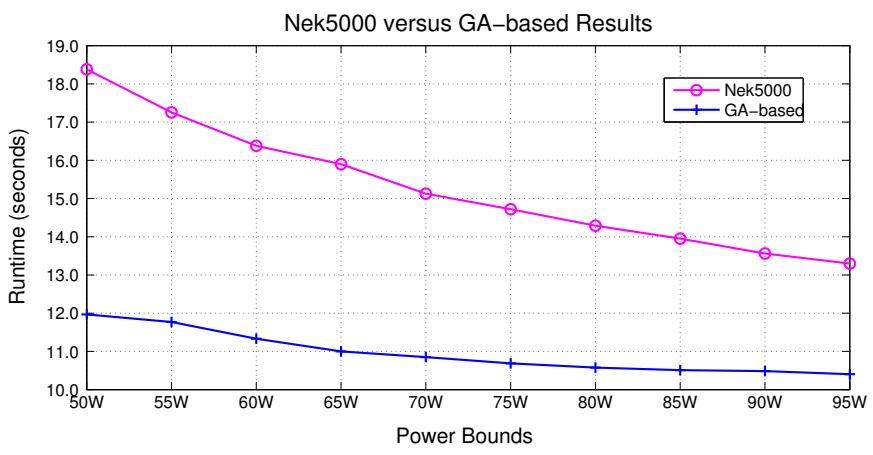

Fig. 13. How runtime changes for $\frac{\partial U}{\partial r}$ calculations using the native implementation in Nek5000 and optimal implementations found using GA driven autotuning for each CPU power bound. The best versions for each power bound have different characteristics for loop permutation and unroll factors.

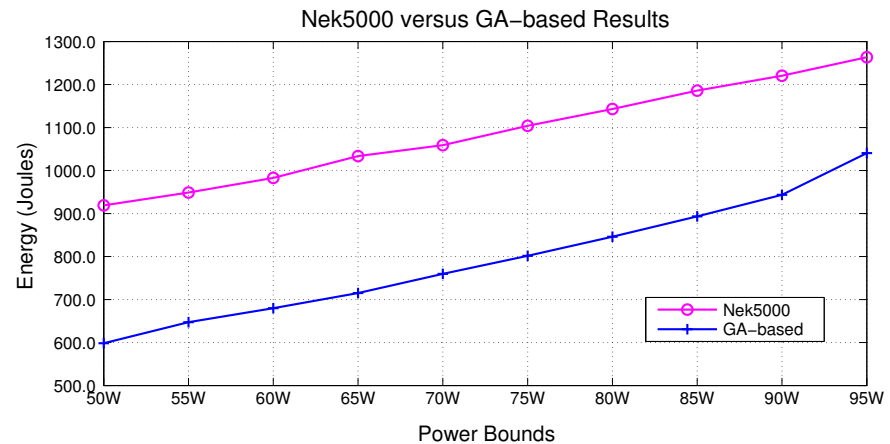

Fig. 14. How energy consumption changes with power bounds for $\frac{\partial U}{\partial r}$ calculations using the native implementation in Nek5000 and optimal implementations found using GA driven autotuning for each CPU power bound. Energy consumption in minimum for the lowest power bound and increases as power bound is increased.

a) Genetic Algorithm Based Results: Perhaps the most interesting and unique study on Intel platform is the behavior of the variants under different power bounds. Without a power bound, the native implementation consumes about $96 \mathrm{~W}$. We present here the results for 10 power bounds ranging between $50 \mathrm{~W}$ and $95 \mathrm{~W}$. The DRAM is not power bounded in these experiments, because this feature is not supported on $\mathrm{Cab}$. Figure 13 shows how runtime drops as power bound is relaxed. The graph compares the performance of the native implementation versus that of the best version found by using the GA algorithm for each power bound. The optimal algorithm was different for each power bound. Thus, the nature of the 


\begin{tabular}{|c|l|l|l|l|l|l|l|l|}
\hline Platforms & $\begin{array}{l}\text { NEK5000 } \\
\text { Time } \\
\text { (seconds) }\end{array}$ & \multicolumn{3}{|c|}{ Exhaustive Autotuning } & \multicolumn{4}{c|}{ GA based Autotuning } \\
\cline { 3 - 9 } & Time & Variants & $\begin{array}{l}\text { \%impro- } \\
\text { ve(nek) }\end{array}$ & Time & Variants & $\begin{array}{c}\text { \%impro- } \\
\text { ve(nek) }\end{array}$ & $\begin{array}{c}\text { \%lower } \\
\text { Perf }\end{array}$ \\
\hline IBM BG/Q & 5.57 & 2.54 & 97716 & 54.3 & 2.58 & 1124 & 53.7 & 1.1 \\
\hline AMD Opteron & 1.81 & 1.02 & 97716 & 43.6 & 1.06 & 1283 & 41.4 & 3.9 \\
\hline AMD Fusion & 1.36 & 0.95 & 32652 & 30 & 0.95 & 485 & 30 & 0.0 \\
\hline Intel Xeon & 18.36 & 11.95 & 5016 & 35 & 11.97 & 120 & 34.8 & 0.5 \\
\hline
\end{tabular}

(a) Performance comparison of derivative operation by platform

\begin{tabular}{|c|l|l|l|l|l|l|l|l|}
\hline Platforms & \multirow{2}{*}{$\begin{array}{l}\text { NEK5000 } \\
\text { Energy } \\
\text { (Joules) }\end{array}$} & \multicolumn{3}{|c|}{ Exhaustive Autotuning } & \multicolumn{4}{c|}{ GA based Autotuning } \\
\cline { 3 - 9 } & Energy & Variants & $\begin{array}{l}\text { \%impro- } \\
\text { ve(nek) }\end{array}$ & Energy & Variants & $\begin{array}{l}\text { \%impro- } \\
\text { ve(nek) }\end{array}$ & $\begin{array}{c}\text { \%lower } \\
\text { Energy }\end{array}$ \\
\hline IBM BG/Q & 292.1 & 131.7 & 96 top & 54.9 & 135 & - & 53.7 & 2.5 \\
\hline AMD Fusion & 17.6 & 11.98 & 32652 & 32 & 12.11 & 485 & 31 & 1.1 \\
\hline Intel Xeon & 918 & 598 & 5016 & 35 & 599 & 120 & 34.7 & 0.17 \\
\hline
\end{tabular}

(b) Energy comparison of derivative operation by platform

Fig. 15. Compute times in seconds and energy consumption in Joules of the derivative computation kernel on different platforms for matrix size $10 \times 10 \times 10$. GA based autotuning has performance and energy consumption very close to exhaustive autotuning. The number of variants used to arrive at the code variants is less than $2 \%$ of the total variants analyzed by the exhaustive search.AMD Fusion numbers pertain to $\frac{\partial U}{\partial r}$ only. Intel Xeon are for matrix size $16 \times 16 \times 16$, and a power bound of $50 \mathrm{~W}$.

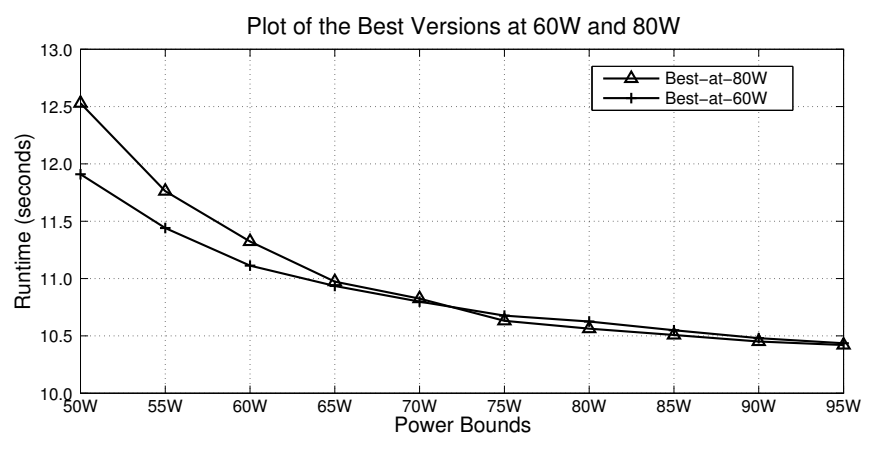

Fig. 16. Comparison of performance for $\frac{\partial U}{\partial r}$ computation for the best algorithm operating at $60 \mathrm{~W}$, versus that operating at $80 \mathrm{~W}$, over a range of power bounds. The plots intersect at about $72 \mathrm{~W}$, showing while one is better for the $50 \mathrm{~W}-72 \mathrm{~W}$ range, the other is better for the $72 \mathrm{~W}-95 \mathrm{~W}$ range, though differences are minor. The GA driven autotuning found different algorithms for each CPU power bound.

optimal algorithm in terms of loop permutation and unroll factors depend on the matrix size as well as the power bound.

Figure 14 shows how energy consumption changes as power bound is relaxed. From this graph we find that operations at lower power bounds is more energy efficient even though they have longer runtime. Thus at higher power bounds, the performance gain obtained by using more power does not compensate for the excess power consumed in the energy equation represented by the product of power consumption and execution time.

Figure 16 shows how the best version of power bounds 60 and $80 \mathrm{~W}$ behave over the range of $50 \mathrm{~W}-95 \mathrm{~W}$. An interesting observation here is that these versions have an intersecting instead of a parallel trend. Though the differences are minor, it also shows that the best algorithm for one power bound is not the same as that for a different power bound and that GA driven autotuning should be run for a new power bound to get the best version when that bound is applied.

Figure 15(a) and (b) summarizes the performance and energy results of the autotuning approaches across the different platforms for matrix size $10 \times 10 \times 10$. We get almost equivalent improvements over NEK5000 when we use GA based autotuning over autotuning based on exhaustive search. The GA based search was off by at most $3.9 \%$ on our tests (from the last columns in Figure 15) compared to exhaustive search, while analyzing far fewer variants.

As mentioned earlier, on IBM BG/Q we did not exhaustively measure power of the variants. Instead we measured power of the top 32 performing variants for each derivative. Hence the variants studied for energy were 96 in all. The variants featured by GA for the best performance on IBM BG/Q were also used to measure power and the energy numbers in Figure 15(b). Also the energy numbers for AMD Fusion pertain to $\frac{\partial U}{\partial r}$ for $N=10$, while those on the Intel platform pertain to $\frac{\partial U}{\partial r}$ computation for $\mathrm{N}=16$, and for a power bound set to $50 \mathrm{~W}$.

Thus, from our experiments on the different platforms, we conclude that the multi-objective optimization problem for energy and performance can be reduced to a two step process optimizing one parameter at each step. For example, in the first step, we could optimize energy and in the process filter the energy efficient variants, and in the second step we analyze the filtered variants to find the best variant for performance. Thus for each matrix size it is possible to come up with at most two best variants, one for energy and the other for performance. Note that the best variant obtained for energy is also a better performing variant and vice versa. Depending 


\begin{tabular}{|l|l|l|l|}
\hline Combination Index & $\begin{array}{l}\text { Cache Size } \\
(\mathrm{KB})\end{array}$ & Line Size (B) & Set Associativity \\
\hline 0 & 2 & 64 & 2 \\
\hline 1 & 8 & 64 & 2 \\
\hline 2 & 8 & 64 & 4 \\
\hline 3 & 8 & 128 & 2 \\
\hline 4 & 8 & 128 & 4 \\
\hline 5 & 16 & 64 & 2 \\
\hline 6 & 16 & 128 & 2 \\
\hline 7 & 16 & 128 & 4 \\
\hline 8 & 16 & 128 & 8 \\
\hline 9 & 16 & 256 & 2 \\
\hline
\end{tabular}

Fig. 17. Table of admissible combinations of cache parameters used in our experiments.

upon the optimization priority for an application one of these variants will be actually linked in the program.

5) gem5 Platform: As mentioned earlier, we used gem5, the well-known micro-architectural simulator, and McPAT, the power estimator, to evaluate different cache configurations for energy and performance trade-offs for out-of-order x 86 cores at the micro-architectural level for derivative computation. The cache parameters considered and their values are given below.

- Cache parameters: For L1 cache, the parameters considered were cache size, line size, set associativity. L1 cache is most relevant for this problem since it involves small matrix multiplications.

\section{- Parameter Values}

- Cache size: $2 \mathrm{~KB}, 8 \mathrm{~KB}, 16 \mathrm{~KB}$

- Cache line size: 64B, 128B, 256B

- Set Associativity: 2, 4, 8

Certain combinations of the above parameter values are rendered inadmissible by gem5 and/or McPAT. The table in Figure 17 gives the admissible combination of values we used for our experiments. Clock speed was $1 \mathrm{GHz}$. We now present results from exhaustive versus genetic algorithm based search.

\begin{tabular}{|l|l|l|l|l|l|l|c|}
\hline Derivatives & $\begin{array}{l}\text { NEK5000 } \\
\text { Time (s) }\end{array}$ & \multicolumn{2}{|c|}{ Exhaustive Autotuning } & \multicolumn{3}{c|}{ GA based Autotuning } \\
\cline { 3 - 8 } & & Time(s) & Variants & $\begin{array}{l}\text { \%impro- } \\
\text { vement }\end{array}$ & Time(s) & Variants & $\begin{array}{c}\text { \%impro- } \\
\text { vement }\end{array}$ \\
\hline dudt & $3.61 \mathrm{e}-04$ & $2.00 \mathrm{e}-04$ & 49560 & 44.6 & $2.03 \mathrm{e}-04$ & 120 & 43.76 \\
\hline dudr & $9.39 \mathrm{e}-05$ & $8.99 \mathrm{e}-05$ & 49560 & 4.4 & $9.00 \mathrm{e}-05$ & 120 & 4.44 \\
\hline duds & $1.28 \mathrm{e}-04$ & $9.50 \mathrm{e}-05$ & 45590 & 25.8 & $9.60 \mathrm{e}-05$ & 120 & 25.00 \\
\hline
\end{tabular}

Fig. 18. Comparison of compute times in seconds, between NEK5000, the best variant using exhaustive autotuning and the best variant found using genetic algorithm based driver.

a) Exhaustive search versus genetic algorithm driven search: We ran exhaustive search for $N=16$ on gem5 to get the best derivative computing codes and the best setting for cache configuration. The total number of code variants analyzed was 4956 for $\frac{\partial U}{\partial r}$ and $\frac{\partial U}{\partial t}$, and 4559 for $\frac{\partial U}{\partial s}$. Each code variant was tried on 10 different cache configurations (Figure 17).

Figure 18 shows a comparison of run times of native NEK5000 implementation, compared to the best versions we get using exhaustive autotuning and GA based autotuning. Performance can be improved by over $40 \%$ compared to the native implementation, using a combination of code transformations and cache configuration. Of particular interest are the GA based autotuning results. The variants identified as the best for

\begin{tabular}{|l|l|l|l|}
\hline Characteristic & dudr & duds & dudt \\
\hline Loop permutation & 123 & 1234 & 123 \\
\hline Loop unroll factors & $1,1,16$ & $1,1,1,16$ & $1,1,16$ \\
\hline Cache size & $16 \mathrm{~KB}$ & $16 \mathrm{~KB}$ & $16 \mathrm{~KB}$ \\
\hline Line size & $64 \mathrm{~B}$ & $64 \mathrm{~B}$ & $64 \mathrm{~B}$ \\
\hline Ways of associativity & 4 & 4 & 4 \\
\hline
\end{tabular}

Fig. 19. Table of characteristics used for NEK5000 code.

\begin{tabular}{|l|l|l|l|}
\hline Characteristic & dudr & duds & dudt \\
\hline Loop permutation & 1234 & 1234 & 1234 \\
\hline Loop unroll factors & $16,1,1,1$ & $16,1,1,1$ & $2,1,2,2$ \\
\hline Cache size & $8 \mathrm{~KB}$ & $16 \mathrm{~KB}$ & $8 \mathrm{~KB}$ \\
\hline Line size & $128 \mathrm{~B}$ & $256 \mathrm{~B}$ & $64 \mathrm{~B}$ \\
\hline Ways of associativity & 4 & 2 & 2 \\
\hline
\end{tabular}

Fig. 20. Table of characteristics for the best performing code using exhaustive autotuning.

each derivative by GA based driver were very close to the optimal variants and these were found after analyzing only about $0.25 \%$ of the total variants analyzed by exhaustive search. The configuration used for the native NEK5000 implementation is shown in Figure 19. Cache configuration was matched up with the existing configuration on HiPerGator, the AMD Opteron platform at UF. NEK5000 uses 4loop-fused implementation for $\frac{\partial U}{\partial r}$ and $\frac{\partial U}{\partial t}$, hence there are only three loops and three unroll factors, for these derivatives, with the innermost loop being completely unrolled. Figures 20 and 21 show the code transformation and cache configuration parameters used to obtain the best variant for the three derivatives using exhaustive autotuning and GA based autotuning, respectively.

Figure 22 shows how changing cache configuration helps in improving performance. We get an additional improvement of $2.5 \%, 1.1 \%$ and $25.7 \%$ upon using a different cache configuration.

Figure 23 shows that energy consumption can be improved by over $40 \%$ compared to the native implementation and Figure 24 shows the code transformation and cache configuration parameters for the optimal variants found using exhaustive search

Figures 25, 26 and 27 show the energy performance characteristics of some variants that are close to the optimal variant for the three derivatives. Each dot in these graphs is a variant representing a unique combination of code transformation parameters for $N=16$ and cache configuration parameters in gem5. Though the best variant (which was identified using exhaustive approach) is indeed the most energy efficient variant for $\frac{\partial U}{\partial r}$ and $\frac{\partial U}{\partial t}$, that is not the case for $\frac{\partial U}{\partial s}$. In fact, for the latter, there are three variants that consume lower energy than the best performing variant as shown in Figure 25. This shows that the best performing code does not necessarily consume the least energy.

\begin{tabular}{|l|l|l|l|}
\hline Characteristic & dudr & duds & dudt \\
\hline Loop permutation & 1234 & 1234 & 2134 \\
\hline Loop unroll factors & $16,1,1,4$ & $16,1,2,1$ & $1,2,1,1$ \\
\hline Cache size & $8 \mathrm{~KB}$ & $16 \mathrm{~KB}$ & $16 \mathrm{~KB}$ \\
\hline Line size & $128 \mathrm{~B}$ & $128 \mathrm{~B}$ & $128 \mathrm{~B}$ \\
\hline Ways of associativity & 4 & 4 & 2 \\
\hline
\end{tabular}

Fig. 21. Table of characteristics for the best performing code using GA based autotuning. 


\begin{tabular}{|l|l|l|l|l|}
\hline Derivatives & \multirow{2}{*}{$\begin{array}{l}\text { NEK5000 } \\
\text { Time }(\mathrm{s})\end{array}$} & \multicolumn{3}{|c|}{ Exhaustive Autotuning } \\
\cline { 3 - 5 } & $3.61 \mathrm{e}-04$ & $2.05 \mathrm{e}-04$ & Time2 $(\mathrm{s})$ & \%improvement \\
\hline dudt & $9.39 \mathrm{e}-05$ & $9.09 \mathrm{e}-05$ & $2.00 \mathrm{e}-04$ & 2.5 \\
\hline dudr & $1.28 \mathrm{e}-04$ & $1.28 \mathrm{e}-04$ & $8.99 \mathrm{e}-05$ & 1.1 \\
\hline duds & \multicolumn{2}{|l}{$9.50 \mathrm{e}-05$} & 25.7 \\
\hline
\end{tabular}

Fig. 22. Comparison of compute times in seconds, between NEK5000, the best variant with the same cache configuration as used for running NEK5000 (Time1), and the best variant taking into consideration all cache configurations (Time2). A change in cache configuration is most effective for duds computation, with a reduction in runtime by $25 \%$

\begin{tabular}{|c|l|c|l|c|c|c|c|}
\hline Derivatives & $\begin{array}{l}\text { NEK5000 } \\
\text { Energy } \\
\end{array}$ & $\begin{array}{l}\text { Energy } \\
(\mathrm{J})\end{array}$ & Variants & $\begin{array}{l}\text { \%impro- } \\
\text { vement }\end{array}$ & Energy(J) & Variants & $\begin{array}{c}\text { \%impro- } \\
\text { vement }\end{array}$ \\
\hline dudt & 0.0134 & 0.00723 & 49560 & 46.00 & 0.00737 & 120 & 45.00 \\
\hline dudr & 0.0034 & 0.00328 & 49560 & 3.44 & 0.00329 & 120 & 3.26 \\
\hline duds & 0.0046 & 0.00350 & 45590 & 23.90 & 0.00353 & 120 & 23.26 \\
\hline
\end{tabular}

Fig. 23. Comparison of energy values in Joules.

\section{CONCLusion}

We have optimized power performance characteristics of one of the most compute intensive kernels of NEK5000 code on a number of platforms using autotuning. We demonstrated how code transformations could aid a more energy efficient version of functionally equivalent code. Compared to NEK5000, we got an average of $34 \%$ improvement in runtime and an average of $37 \%$ improvement in energy on AMD Fusion over different matrix sizes. On AMD Opteron, we got an average of $28 \%$ improvement in runtime over different matrix sizes and on IBM BG/Q an average of 53.7\% improvement in runtime and energy consumption for matrix size $10 \times 10 \times 10$. Our work highlights the importance of autotuning as a platform independent optimization strategy that relieves developers from manually tuning their codes on complex architectures.

To address the limitations of an exhaustive approach, we demonstrate how genetic algorithms can be used to efficiently navigate through the configuration space. In our comparison study of the exhaustive autotuning versus autotuning driven by genetic algorithm, we found that the best variants found by the latter performed within $3.9 \%$ of the performance of the optimal variant found by the exhaustive search. The number of variants evaluated by the genetic algorithm based driver was only about $1.49 \%$ of the total variants evaluated during the exhausting search. Genetic algorithm based autotuning approach also helps us to perform multi-objective optimization even when optimization objectives are conflicting by setting an appropriate fitness function. In this work, our energy benchmarking shows that there is a strong correlation between performance and energy requirements. Thus, for the target rou-

\begin{tabular}{|l|l|l|l|}
\hline Characteristic & dudr & duds & dudt \\
\hline Loop permutation & 1234 & 1234 & 1234 \\
\hline Loop unroll factors & $16,1,1,1$ & $16,1,1,1$ & $2,1,2,2$ \\
\hline Cache size & $8 \mathrm{~KB}$ & $8 \mathrm{~KB}$ & $8 \mathrm{~KB}$ \\
\hline Line size & $128 \mathrm{~B}$ & $128 \mathrm{~B}$ & $64 \mathrm{~B}$ \\
\hline Ways of associativity & 4 & 4 & 2 \\
\hline
\end{tabular}

Fig. 24. Table of characteristics for the most energy efficient code.

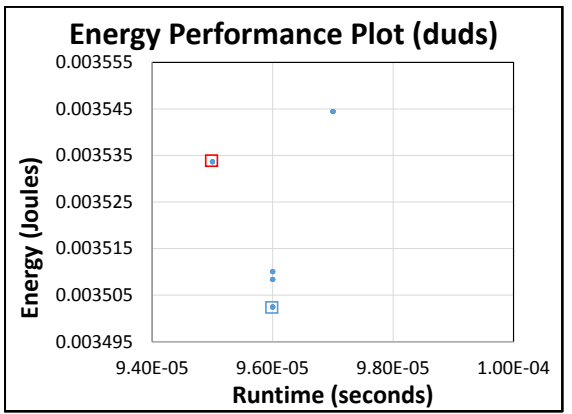

Fig. 25. Energy performance plot for the different variants close to the optimal variant for duds. The performance optimal variant is enclosed by a red box, whereas the most energy efficient version is enclosed in a blue box.

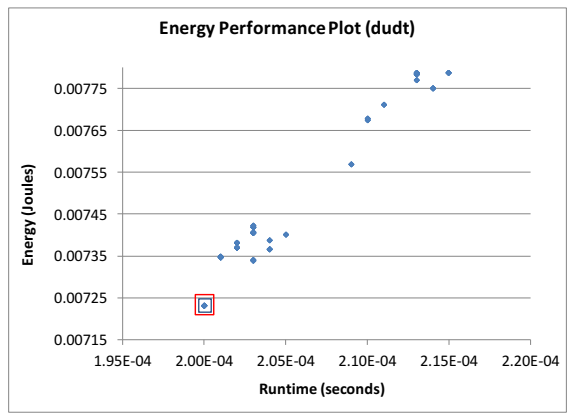

Fig. 26. Energy performance plot for the different variants close to the optimal variant for dudt. The same variant is performance and energy optimal.

tines, improving performance result in simultaneous reduction in energy requirements.

Genetic algorithms are also amenable to complex objective functions that take performance and energy both into account for optimization. Additionally, they can be easily modified to include architectural parameters such as the line size and associativity of a cache to effectively explore a hardware software co-design. Both of these areas will be part of our future work.

\section{ACKNOWLEDGMENT}

We would like to thank members of the Center for Compressible Multiphase Turbulence at the University of Florida for their valuable feedback throughout the work. We are also grateful to David DeBonis, James Laros and others at the Sandia National Laboratory for their help in using PowerInsight. We express our gratitude to Barry Rountree and Scott Walker at Lawrence Livermore National Laboratory for their help with measuring and clamping power using RAPL. This work was funded by the U.S. Department of Energy, National Nuclear Security Administration, Advanced Simulation and Computing Program, as a Cooperative Agreement under the Predictive Science Academic Alliance Program, Contract No. DOE-NA0002378.

\section{REFERENCES}

[1] J. H. Holland, Adaptation in natural and artificial systems: An introductory analysis with applications to biology, control, and artificial intelligence, University of Michigan Press, 1975. 


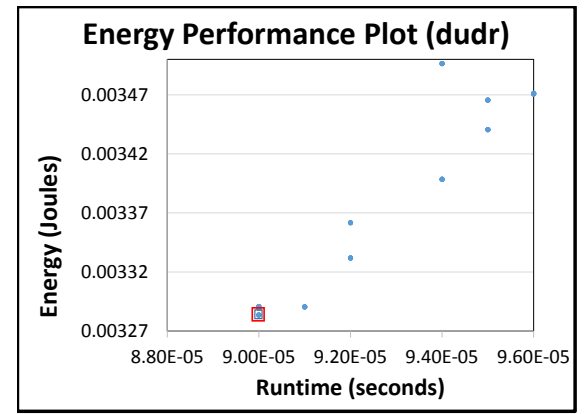

Fig. 27. Energy performance plot for the different variants close to the optimal variant for dudr. The same variant is both performance and energy optimal and is enclosed in red and blue boxes.

[2] P. Fischer, J. Lottes, S. G. Kerkemeier, NEK5000 Web page, http://nek5000.mcs.anl.gov, 2008.

[3] DOE, Architectures and technology for extreme scale computing, December 2009

[4] T. Banerjee and S. Ranka, A Genetic Algorithm based Autotuning Approach for Performance and Energy Optimization, IEEE International Green and Sustainable Computing 2015

[5] A. Tiwari, C. Chen, J. Chame, M. Hall and J. Hollingsworth, A Scalable Autotuning Framework for Compiler Optimization, IEEE IPDPS, May, 2009.

[6] J. H. Laros, III, P. Pokorny, and D. DeBonis, PowerInsight A Commodity Power Measurement Capability, The Third International Workshop on Power Measurement and Profiling in conjunction with IEEE IGCC 2013, 2013.

[7] J. Shin, M. W. Hall, J. Chame, C. Chen, P. F. Fischer, P. D. Hovland, Speeding Up Nek5000 with Autotuning and Specialization ACM International Conference on Supercomputing, 2009

[8] J. Shin, M. W. Hall, J. Chame, C. Chen, P. D. Hovland, Autotuning and specialization: Speeding up matrix multiply for small matrices with compiler technology. International Workshop on Automatic Performance Tuning, October, 2009.

[9] C. Chen, J. Chame, M. W. Hall, CHiLL: A Framework for Composing High-Level Loop Transformations, Technical Report 08-897, University of Southern California, CS Dept, 2008.

[10] R. Bertran, Y. Sugawara, H. M. Jacobson, A. Buyuktosunoglu, P. Bose, Application-level power and performance characterization and optimization on IBM Blue Gene/Q systems, IBM Journal of Research and Development, 57, 1-2, 2013.

[11] S. Wallace, V. Vishwanath, S. Coghlan, Z. Lan, M. E. Papka, Measuring Power Consumption on IBM Blue Gene/Q, IEEE International Symposium on Parallel and Distributed Processing Workshops, 2013.

[12] E. A. Leon, I. Karlin, Characterizing the Impact of Program Optimizations on Power and Energy for Explicit Hydrodynamics, International Parallel and Distributed Processing Symposium, 2014.

[13] R. C. Whaley, A. Petitet, J. Dongarra, Automated Empirical Optimizations of Software and the ATLAS project, Parallel Computing, 27, 1-2, pp. 3-35, 2001.

[14] R. C. Whaley, J. Dongarra, Automatically Tuned Linear Algebra Software, ACM/IEEE Conference on Supercomputing 1998.

[15] R. Vuduc, J. W. Demmel, K. A. Yelick, OSKI: A library of automatically tuned sparse matrix kernels, Proceedings of the Scientific Discovery through Advanced Computing Conference, June, 2005.

[16] J. Bilmes, K. Asanovic, C-W. Chin, J. Demmel, Optimizing matrix multiply using PHiPAC: a portable, high-performance, ANSI coding methodology, Proceedings of the ACM International Conference on Supercomputing 1997.
[17] K. Sharma, I. Karlin, J. Keasler, J. McGraw, V. Sarkar, UserSpecified and Automatic Data Layout Selection for Portable Performance, Technical Report, LLNL-TR-637873 May, 2013

[18] H. Maini, K. Mehrotra, C. Mohan and S. Ranka, KnowledgeBased Nonuniform Crossover, Proceedings of the First IEEE Conference on Evolutionary Computation, pp.22-27, Jun 1994.

[19] I-H. Chung, J. K. Hollingsworth, Using information from prior runs to improve automated tuning systems, ACM/IEEE SuperComputing 2004.

[20] E. Cantu-Paz, A Survey of Parallel Genetic Algorithms $C A L-$ CULATEURS PARALLELES Vol. 10, 1998.

[21] C. Notredame, D. G. Higgins, SAGA: Sequence Alignment by Genetic Algorithm, Nucleic Acid Research, Oxford Journals, Vol. 24, Issue 8, pp.1515-1524, April 1996.

[22] Y. Li, K. H. Ang, G. Chong, W. Feng, K. Tan, H. Kashiwagi, CAutoCSD-evolutionary search and optimisation enabled computer automated control system design, International Journal of Automation and Computing, 1, 1, pp 76-88, October, 2004.

[23] T. C. Smith and I. H. Witten, Learning Language using Genetic Algorithms, Lecture Notes in Computer Science, Vol. 1040, pp 132-145, 1996.

[24] J. Dongarra, H. Ltaief, P. Luszczek, and V. M. Weaver, Energy footprint of advanced dense numerical linear algebra using tile algorithms on multicore architecture, CGC, 2012.

[25] R. Joseph and M. Martonosi, Run-time power estimation in high performance microprocessors. Proceedings of the 2001 International Symposium on Low Power Electronics and Design, 2001.

[26] G. Contreras and M. Martonosi, Power prediction for intel xscale reg; processors using performance monitoring unit events. Proceedings of the 2005 International Symposium on Low Power Electronics and Design, 2005.

[27] B. Goel, S. McKee, R. Gioiosa, K. Singh, M. Bhadauria, and M. Cesati, Portable, scalable, per-core power estimation for intelligent resource management, IGCC, 2010.

[28] K. Singh, M. Bhadauria, and S. A. McKee, Real time power estimation and thread scheduling via performance counters. SIGARCH Computer Architecture News, 37,2, pp:46-55, July 2009.

[29] Intel, Intel 64 and IA-32 Architectures Software Developer's Manual Volume 3A, 3B, and 3C: System Programming Guide, Parts 1 and 2, 2011.

[30] M. McFadden, K. Shiga, B. Rountree, GitHub scalabilityllnl/msr-safe, 2015. https://github.com/scalability-llnl/msr-safe

[31] S. Walker, K. Shoga, B. Rountree, L. Morita, GitHub scalability-llnl/libmsr, 2015. https://github.com/scalability1ln1/libmsr (Accessed on 10/12/2015)

[32] N. Binkert, B. Beckmann, G. Black, S. K. Reinhardt, A. Saidi, A. Basu, J. Hestness, D. R. Hower, T. Krishna, S. Sardashti, R. Sen, K. Sewell, M. Shoaib, N. Vaish, M. D. Hill, and D. A. Wood, The gem5 simulator, SIGARCH Computer Architecture News, 39(2):1-7, August 2011.

[33] S. Li, J. H. Ahn, R. D. Strong, J. B. Brockman, D. M. Tullsen, and N. P. Jouppi, The McPAT framework for multicore and manycore architectures: Simultaneously modeling power, area, and timing, ACM Transactions of Architecture and Code Optimization, 10(1):5:1-5:29, Apr. 2013

[34] F. A. Endo, D. Courousse and H-P. Charles, Micro-architectural simulation of embedded core heterogeneity with gem5 and McPAT, RAPIDO, 2015

[35] T. Blickle, J. Teich, and L. Thiele, System-level synthesis using evolutionary algorithms, Design Automation for Embedded Systems, 3(1), pp. 23-58, 1998.

[36] R. P. Dick and N. K. Jha, MOGAC: A multiobjective genetic algorithm for hardware-software co-synthesis of distributed embedded systems, IEEE Transactions Computer-Aided Design, pp. 920-935, Oct. 1998. 
[37] T. Givargis, F. Vahid, and J. Henkel, System-level exploration for pareto-optimal configurations in parameterized system-on-achip, IEEE Transactions on VLSI Systems, vol. 10, pp. 416-422, 2002.

[38] T. Givargis and F. Vahid, Platune: A tuning framework for system-on-a-chip platforms, IEEE Transactions on ComputerAided Design vol. 21, pp. 1317-1327, 2002.

[39] G. Ascia, V. Catania, and M. Palesi, A GA-based design space exploration framework for parameterized system-on-a-chip platforms, IEEE Transactions on Evolutionary Computation, vol. 8, pp. 329-346, 2004.

[40] B. Rountree, D. H. Ahn, B. R. de Supinski, D. K. Lowenthal and M. Schulz, Beyond DVFS: A First Look at Performance Under a Hardware-Enforced Power Bound, IPDPS 2012. 